\title{
REGARD SUR LA RECHERCHE OPHTALMOLOGIQUE
}

\section{Yves Pouliquen}

Directeur de l'unité de recherche d'ophtalmologie, Inserm $U$ 86, HôtelDieu, 1 Place du parvis Notre-Dame, 75181 Paris cedex 04.

\section{RÉFÉRENCES}

1. Vision Research, a National Plan, the 1983 Report of the National Advisory Eye Council, Bethesda, MD : National Institute of Health, 1983.

2. Réduire les Handicaps, rapport es maladies et altérations de la vision sont parmi les affections les plus douloureuses pour l'individu, l'une des composantes essentielles des maladies du troisième âge et une très lourde charge pour la société. Si l'on se réfere aux États-Unis où l'on dispose de statistiques précises, 10 millions de personnes de ce pays souffrent d'une vision défectueuse ne pouvant être corrigée par des lunettes ou des lentilles, dont 1,5 million ne peuvent lire le journal, et 0,5 million sont aveugles. Le coût annuel des maladies oculaires y est estimé à plus de 14 milliards de dollars [1].

Cette prise de conscience a largement contribué à développer le budget de recherche américain sur l'œil et la vision, et a attiré vers la recherche ophtalmologique un grand nombre de chercheurs. C'est ainsi que l'Association for Research in Vision and Ophthalmology (ARVO) compte près de 4000 membres. Son congrès annuel réunit en de multiples sessions simultanées toutes les disciplines biologiques impliquées dans la recherche ophtalmologique. Un rapport sur les handicaps visuels, réalisé par l'Inserm [2], indique des chiffres proportionnellement similaires sur l'épidémiologie des maladies oculaires en France. La recherche ophtalmologique se développe en Europe, où l'Association for Eye Research (AER) rassemble à une échelle beaucoup plus modeste les chercheurs et médecins intéressés par l'œil et la vision.

L'effort de recherche a porté d'abord sur l'appareillage et les techniques d'exploration fonctionnelle, d'imagerie et de chirurgie de l'œil, dont les progrès spectaculaires sont à l'origine des succès thérapeutiques actuels. Ce développement technologique et clinique ne représente toutefois qu'une part de l'ensemble de la recherche en ophtalmologie. Les voies de la recherche biologique sur l'œil et la vision sont aussi prometteuses. La physiologie et la pathologie de l'œil offrent au biologiste un champ d'action considérable.

L'œil est un organe relativement clos, composé de nombreux tissus différents dont les fonctions complémentaires assurent la fonction visuelle (figure 1). Par la variété de ses composants, ce microcosme est siège de multiples phénomènes physiologiques et est impliqué dans les maladies les plus variées. Aussi les recherches sur l'œil sont-elles très diversifiées.

Dans de nombreux domaines de la recherche biologique et médicale, l'œil présente des avantages pour l'étude de processus généraux. L'un d'eux est l'accessibilité de cet organe à l'observation directe de ses structures internes, liée à la transparence de ses milieux : cornée, cristallin, vitré. On peut observer in vivo, par exemple, les vaisseaux et leur pathologie dans la rétine (rétinophotographíe), ou la couche cellulaire endothéliale de la cornée à une échelle semblable à celle de l'histologie (microscopie spéculaire). De multiples modèles animaux permettent d'étudier par l'observation directe les processus de l'inflammation, la néovascularisation, la cicatrisation. Grâce à l'angiofluorométrie, on étudie les facteurs de perméabilité des barrières endothéliales et épithéliales (barrière hémato-oculaire).

La constitution des tissus oculaires en feuillets dissociables facilite la purification de ces tissus, nécessaire à des travaux de biologie cellulaire et de biochimie. Quatre épithéliums sont aisément mis en culture pure et utilisés notamment en pharmacologie cellulaire. Le cristallin, les éléments photorécepteurs de la rétine (segments externes des bâtonnets) sont des matériels biologiques relativement simples, favorables à l'analyse biochimique.

En pathologie, les thèmes de recherche incluent les vasculopathies (rétinopathie diabétique, vasculites inflammatoires), les neuropathies, la pathologie du tissu conjonctif (maladies de la cornée et de la sclère), la sénescence (maculopathie, cataracte), les maladies tumorales (mélanome, rétinoblastome), l'inflammation 
d'origine virale (herpès), bactérienne, parasitaire (toxoplasmose), l'immunopathologie sous ses différentes formes : allergie, auto-immunité, immunologie anti-infectieuse ou de greffe (cornée).

$\mathrm{La}$ rédaction de médecine/sciences a choisi d'évoquer dans les articles qui suivent la variété des études dont l'œil peut être l'objet, tout en exprimant une recherche d'intérêt général : ainsi l'œil offret-il des champs d'étude fondamentale en biologie cellulaire avec la phototransduction, en neurophysiologie avec les neurotransmetteurs et en immunopathologie avec l'uvéo-rétinite auto-immune. La photo-transduction, qui assure, dans le segment externe de la cellule photoréceptrice, l'amplification du signal lumineux capté par un récepteur, le photopigment, et sa traduction en influx nerveux, est un des modèles les plus utiles à l'analyse de la transduction membranaire. Les segments externes sont aisément isolés et purifiés. Ils contiennent un petit nombre de protéines bien caractérisées par les méthodes électrophorétiques et immunochimiques. Les possibilités de reconstituer in vitro des systèmes élémentaires par l'interaction de protéines purifiées et la maniabilité du stimulus, la lumière, sont des avantages mis à profit pour comprendre la perception lumineuse, mais aussi les mécanismes de la transduction en général. L'étude de la phototransduction donne aussi l'espoir d'identifier les déficits responsables des maladies dégénératives des photorécepteurs, souvent génétiquement transmises, qui sont une cause essentielle de cécités chez les sujets jeunes.

Certaines des protéines des photorécepteurs sont aussi des autoantigènes qui suscitent des maladies auto-immunes de l'œil. Le modèle de l'uvéo-rétinite autoimmune est favorable à l'étude des mécanismes de l'autoimmunité spécifique d'organe. De nombreuses maladies humaines inflammatoires ou dégénératives de la rétine et de l'uvée (uvéites, $\mathrm{m} / \mathrm{s} n^{\circ} 4$ ool. 3, avril 87 rétinopathies) mettent en jeu cette auto-immunité. L'identification des structures moléculaires responsables de l'auto-antigénicité et le développement des méthodes d'immunorégulation donnent l'espoir de proposer des remèdes à ces maladies.

Prolongement du système nerveux central, la rétine, dans ses systèmes de transmission et d'amplification, obéit à la fois aux règles générales de la neurobiologie et aux siennes propres. L'application à la rétine des techniques récentes de mise en évidence des neurotransmetteurs qu'utilisent les neurones pour véhiculer les messages, permet d'explorer l'admirable et complexe réseau de ses trois étages de neurones. Le chercheur doit dresser l'inventaire des terminaisons neuronales, en re- constituer l'architecture et établir leurs relations, superposer les messages chimiques à l'électrophysiologie afin de saisir les règles du fonctionnement de cet ensemble. Cet ensemble code la perception lumineuse en contrastes, reliefs, couleurs, directions, mouvements, autant de messages acheminés vers le cerveau pour l'élaboration d'une image.

Tout est dans l'œil, ce qui donne à cette introduction le ton d'un inventaire. Espérons que nous serons pardonnés de l'avoir imposé si nous avons pu rendre sensible aux lecteurs l'intérêt de ce petit organe dont la représentation est si vaste en notre cerveau, et l'image si chère au poète...

"En un sens l'œil contrebalançait l'abîme " (M. Yourcenar - L'cuvre au noir)

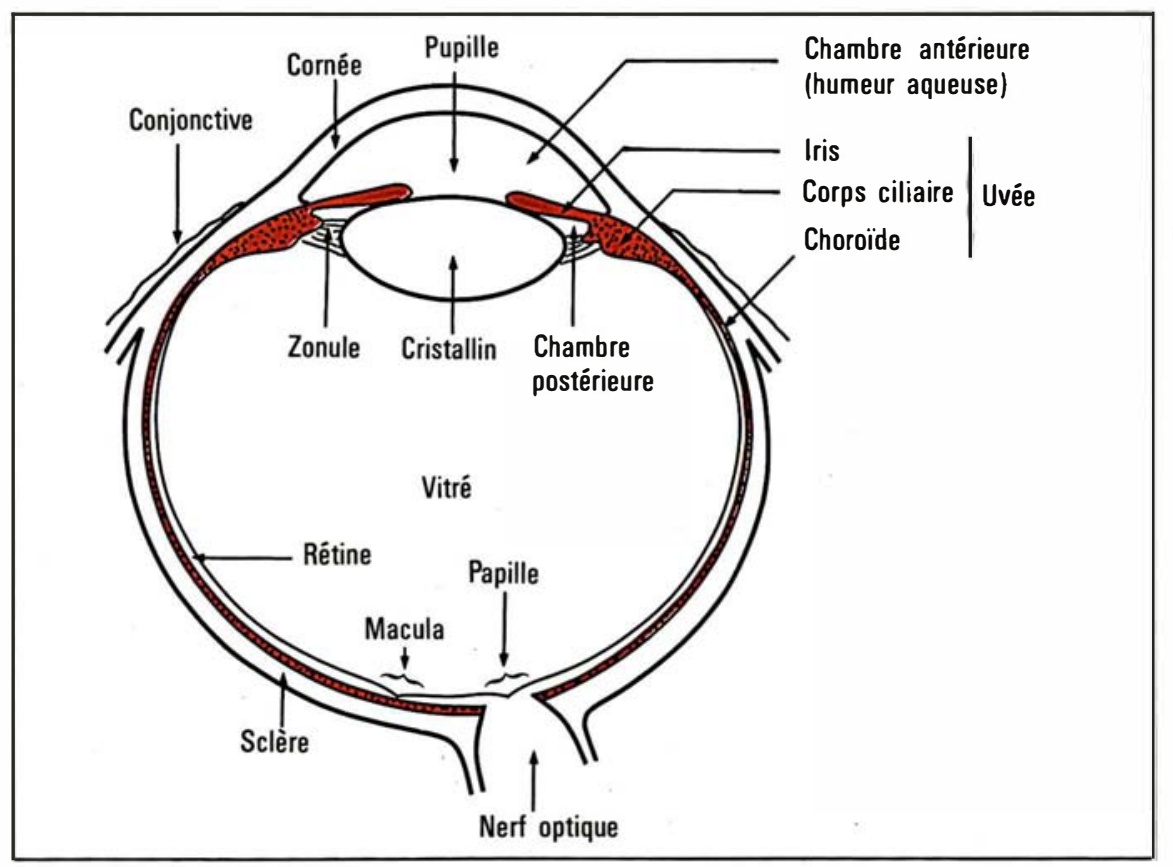

Figure 1. Coupe horizontale de l'coil. L'œil est limité par une coque très résistante, la sclère, dont la partie antérieure, transparente, forme la cornée. Les éléments optiques de l'œil, la cornée et le cristallin focalisent les images sur la rétine, dépendance du système nerveux central qui forme une mince couche photosensible à la face interne de la paroi oculaire, dans sa partie postérieure. L'uvée, tissu conjonctif pigmenté et très richement vascularisé, comporte trois parties : 1 . la choroïde sépare la rétine de la sclère; 2 . le corps ciliaire a deux fonctions : sécréter l'humeur aqueuse qui nourrit le cristallin et la cornée, et, par l'intermédiaire des fibres de la zonule, maintenir le cristallin à sa place et modifier sa forme (accommodation); 3 . I'iris règle la quantité de lumière qui atteint la rétine en changeant l'ouverture de la pupille. Le vitré est un gel transparent qui occupe la plus grande partie du volume de l'œil. 